\title{
Méthode pour caractériser l'irrégularité de la forme des tiges en section transversale et son évolution au cours du temps
}

\author{
JP Bouillet \\ Mission CIRAD-Forêt, BP 745, Antananarivo, Madagascar
}

(Reçu le 12 septembre 1991; accepté le 25 septembre 1992)

\begin{abstract}
Résumé - La section transversale des tiges est souvent assimilée à un disque parfait ou quasiparfait, alors que cela reste en fait une exception. Diverses définitions sont classiquement données pour définir l'«excentricité» d'une tige, mais aucune d'elles ne permet de caractériser assez précisément le centre géométrique de la section et son évolution au cours du temps, ce qui est le but de cet article. L'“excentricité» recouvre l'excentricité en elle-même, non-concordance entre centre géométrique de la section et moelle de l'arbre; le méplat, aplatissement de la section résultant de la croissance privilégiée dans une direction donnée. La caractérisation de l'excentricité est basée sur l'assimilation des accroissements radiaux annuels à des vecteurs et sur l'évolution de leur somme. On peut connaître ainsi l'excentricité résultant de l'apparition de chaque nouvel accroissement annuel et l'évolution du centre géométrique de la section depuis l'origine. On peut rapprocher les observations faites à un même niveau pour différentes tiges, à différents niveaux d'une même tige et sur des arbres de vigueurs différentes. Le méplat est caractérisé. L'utilisation de diamètres passant par la moelle est préconisée si le nombre de rayons étudiés est assez élevé $(\geq 16)$.
\end{abstract}

excentricité / méplat / méthodologie / forme des arbres

Summary - A method to characterize irregularity in the cross-sectional form of a stem and its evolution with time. The section perpendicular to the stem axis (SPSA) is often considered to be a perfect or almost perfect disk, although in fact this case is exceptional.

The aim of this study was to characterize the eccentricity of SPSA by estimating its geometric center and its evolution with time. Eccentricity includes:

- eccentricity itself, ie no concordance between the geometric center of SPSA and the pith;

- flattening of the SPSA due to a greater increment in a given direction.

Eccentricity is characterized by identifying several radial increments to vectors and the evolution of their sum. It is therefore possible to characterize eccentricity resulting from each year of growth and the evolution of the geometric center of SPSA from the beginning. It is also possible to link observations made at the same height for different trees, at different heights for a given tree and for trees with different vigors. Flattening can be accurately characterized by diameters passing through the pith if the number of radii is equal to at least 16.

eccentricity / flattening / methodology / bole form 


\section{INTRODUCTION}

Plusieurs études prenant en compte le problème de la forme des arbres retiennent a priori que la section transversale de la tige aux différents niveaux du tronc (notée section dans la suite de l'article) est un disque parfait.

Cette hypothèse est présente dans de nombreux travaux se rapportant au profil en long des tiges qui donnent classiquement le diamètre, supposé constant, quelle que soit l'orientation selon laquelle il est pris, comme une fonction de la hauteur (Cailliez, 1980; McClure et al, 1986; Demaerschalk et al, 1977; Kozak, 1988; Farrar, 1987; Lowell, 1986; Gordon et al, 1986; M'Hirit et al, 1983; Armitage et al, 1980).

D'autres travaux consacrés plus particulièrement à l'étude de la répartition de la surface des cernes annuels le long du tronc supposent implicitement que cette hypothèse est vérifiée (Mitchell, 1975; Mitchell et al, 1972; Farrar, 1961; Larson, 1963).

Mais, en fait, une section en forme parfaite ou quasi parfaite de disque reste souvent l'exception, comme l'ont montré par exemple Williamson (1975) et Monserud (1979) sur Pseudotsuga menziesii, Kellog et Barber (1981) sur Tsuga heterophylla, Daniels et Schutz (1975) sur Pinus patula ou Biging et Wensel (1988) sur différentes espèces de conifères poussant en mélange; de la même façon, l'excentricité parfois fortement prononcée de Pinus pinaster dans le massif des Landes en France est un fait bien connu (Polge et llly, 1967).

Cependant, même si l'on admet que les arbres puissent présenter une excentricité, il convient de la caractériser, afin de pouvoir en tenir compte ultérieurement.

\section{CARACTÉRISATION GÉNERALE DE L'EXCENTRICITÉ}

L'«excentricité»** d'une tige notée $E$ peut être définie de plusieurs manières. Ainsi, en se référant à la figure 1 , on peut poser que l'excentricité est définie comme :

- le rapport du plus grand rayon à celui qui lui est opposé (Polge et llly, 1967) : $E_{1}=$ $R / r$; il faut remarquer que le rayon opposé au plus grand n'est pas forcément le plus petit, par exemple ici $r 1<r$;

- le rapport entre le diamètre perpendiculaire au plus grand diamètre et ce dernier (Monserud, 1979; Biging et Wensel, 1988) : $E_{2}=M=d / D$; il est à remarquer que Polge et llly (1967) définissent ce rapport comme caractérisant le méplat $\mathrm{M}$ de la section;

- le rapport de la différence entre le plus grand diamètre et le diamètre perpendiculaire à ce dernier diamètre (Williamson, 1975) : $E_{3}=(D-d) / d=D / d-1=1 / E_{2}-1$;

- le rapport du plus petit diamètre au plus grand (Kellog et Barber, 1981): $E_{4}=d^{\prime} / D$; toutes ces mesures s'entendent sous écorce, mais il est évident qu'il serait possible aussi de définir une excentricité sur écorce.

La multiplicité des définitions utilisées montre que la notion d'excentricité n'est pas toujours facile à quantifier précisément. Cette situation est préoccupante dans la mesure où ce phénomène a des répercussions sur les propriétés technologiques du bois. En effet, il est classiquement avancé que l'excentricité d'une tige s'accompagne de la formation de bois de réaction (de compression chez les rési-

\footnotetext{
* Souvent équation du type $d / D 1,30 m=f(h / H T)$. $d$ : diamètre de la tige à une hauteur $h ; D 1,30 m=$ diamètre de la tige à 1,30 m et HT : hauteur totale de la tige.

** Traduction de l'anglais eccentricity.
} 


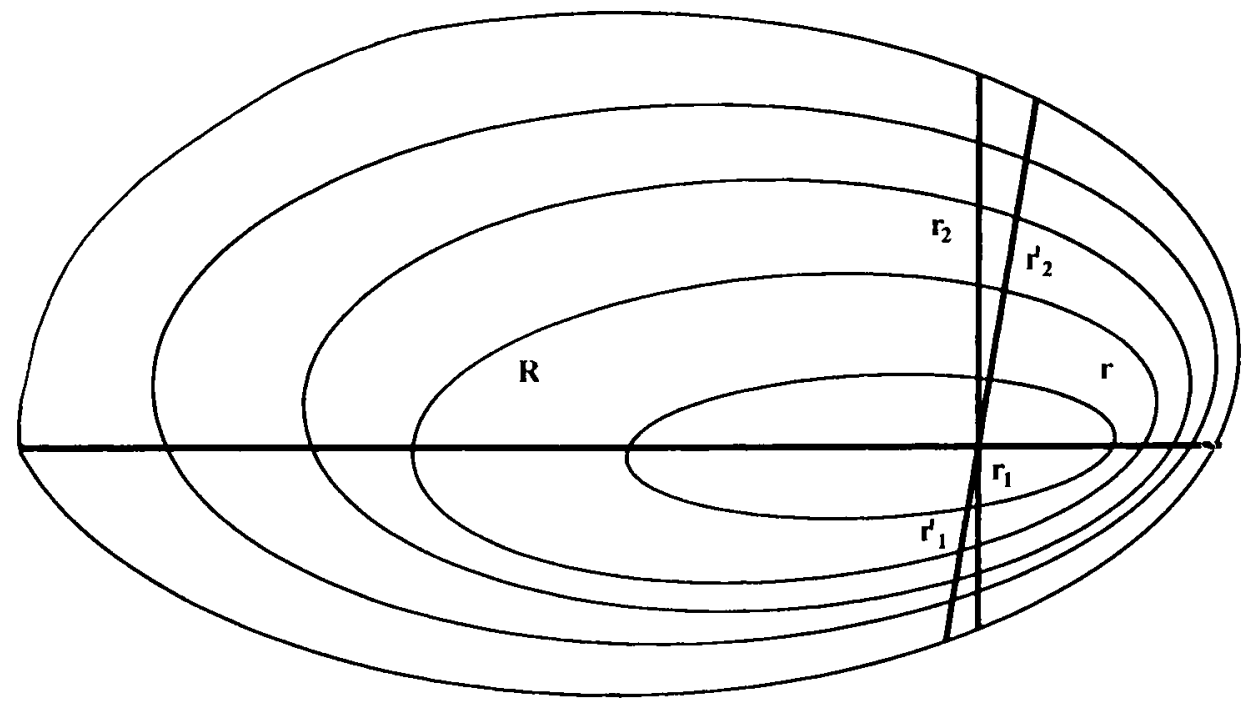

Fig 1. Section transversale de tige présentant une excentricité. $R+r=D ; r_{1}+r_{2}=d ; r_{1}^{\prime}+r_{2}^{\prime}=d$.

neux, de tension chez les feuillus) (Coue et al, 1990; Fournier, 1989; Détienne, 1976; Wilson et Archer, 1983). Or ce type de bois présente des caractéristiques différentes de celles du bois normal et conduit à l'obtention de produits aux qualités technologiques inférieures (Coue et al, 1990; Fournier, 1989; Détienne, 1976). II est donc important d'obtenir des arbres à faible excentricité et, en corollaire, de pouvoir quantifier l'impact éventuel des facteurs du milieu (traitements sylvicoles, vent, température...) sur ce phénomène.

Les définitions précédentes ne permettent pas de répondre à cet objectif. En effet, seules 2 directions sont utilisées pour caractériser l'irrégularité de la section. Une même valeur d'indice peut donc recouvrir des formes de section sensiblement différentes (voir fig 2 par rapport à la définition de $E_{2}$ et $E_{3}$ ). De plus, le classement des individus en fonction de leur ex-

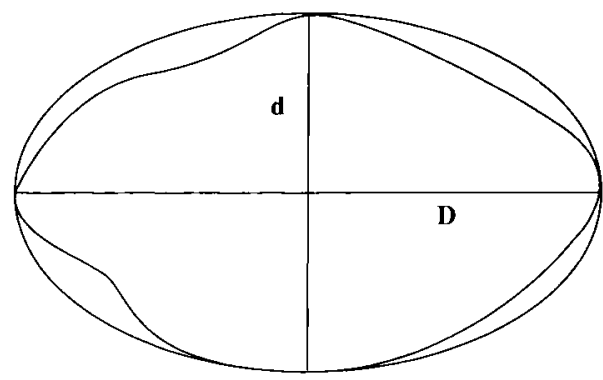

Fig 2. Exemple de 2 sections de formes différentes, mais présentant un indice d'excentricité $\mathrm{d} / \mathrm{D}$ égal.

centricité peut varier suivant les indices employés (fig 3).

Ajoutons que les auteurs auxquels nous faisons référence ne se préoccupent que de la caractérisation de l'état final et que 


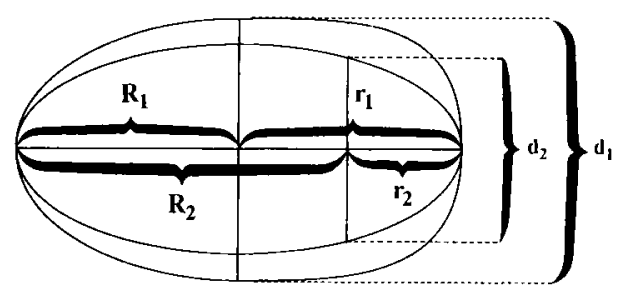

$\frac{d_{1}}{R_{1}+r_{1}}>\frac{d_{2}}{R_{2}+r_{2}}$ mais $\frac{R_{1}}{r_{1}}<\frac{R_{2}}{r_{2}}$

Fig 3. Exemple de 2 sections présentant un classement d'excentricité différent suivant le type d'indice choisi.

les différentes définitions proposées ne facilitent pas l'étude de l'évolution de l'excentricité, dans la mesure où les différents paramètres sollicités (plus grand diamètre, plus grand rayon) peuvent changer de support tout au long de la croissance de l'arbre.

En dernier lieu, il apparaît que ces paramètres tentent de recouvrir 2 notions :

- l'excentricité proprement dite, c'est-àdire la non-concordance entre centre géométrique de la section et moelle de l'arbre;

- le méplat caractérisant l'aplatissement de la section, dû à la croissance moindre sur un diamètre par rapport au diamètre perpendiculaire (passage du périmètre de la section d'une forme générale circulaire à une forme générale d'ellipse).

Il est essentiel de distinguer ces 2 notions, comme l'a indiqué Pawsey (1966); Polge et Illy (1967) en ont tenu compte en proposant 2 indices différents; une section peut, par exemple, présenter une forte excentricité tout en étant en forme de disque parfait et une autre aucune excentricité mais un méplat important (périmètre en forme d'ellipse) (fig 4).

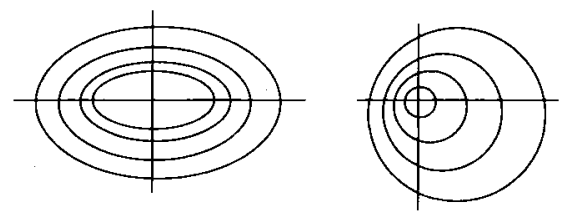

Fig 4. Section en forme de disque parfait mais très excentrée et section très méplate mais centreee.

En fait, pour une section donnée, il apparaît utile de pouvoir : "définir" un point se rapprochant le plus possible du centre géométrique de la section; cela est nécessaire pour caractériser avec assez de précision le vecteur moelle - centre géométrique; et de rendre compte de l'évolution de l'excentricité au cours de la croissance de l'individu.

Afin de répondre à la première exigence, un certain nombre d'auteurs tentent d'assimiler les sections des tiges à des surfaces dont il est aisé de connaître le centre géométrique.

Par exemple, Boissieras (1984) considère ainsi la section de Pinus pinaster comme étant celle d'une ellipse, dont sont déterminés expérimentalement le grand axe qui passe, par hypothèse, par la moelle de la tige, puis le petit axe perpendiculaire passant par le milieu du grand axe.

Cependant, il faut souligner que la section des arbres n'est évidemment jamais rigoureusement assimilable à une surface régulière, et que cette assimilation peut entraîner un biais important dans l'évaluation des aires des sections, ou de l'accroissement en surface terrière produit sur une période donnée (Biging et Wensel, 1988).

II apparaît donc souhaitable de pouvoir proposer une méthode relativement aisée 
à mettre en œuvre, et permettant de caractériser avec assez de précision l'anisotropie radiale d'une section à un instant donné, et son évolution au cours du temps.

\section{EXCENTRICITÉ}

La méthode que nous proposons est fondée sur l'assimilation des accroissements radiaux annuels à des vecteurs et sur l'évolution de leur somme.

Cette idée a été avancée par Marutani et al (1987), dont les travaux servent de base à la présente étude.

\section{Principes de la méthode proposée par Marutani et al}

Soit une rondelle prélevée perpendiculairement à l'axe de la tige et, sur le plan de cette rondelle, un repère $(0, \vec{i}, \vec{j})$ où 0 est la moelle de l'arbre et $\vec{i}$ et $\vec{j}, 2$ vecteurs unitaires orthogonaux. Les accroissements radiaux annuels sur chacun des 4 demiaxes associés à $\vec{i}$ et $\vec{j}$ peuvent être représentés par les vecteurs $\vec{a}_{t}, \vec{b}_{t}, \vec{c}_{t}$ et $\vec{d}_{t}$ (fig 5). La résultante $\vec{l}_{t}$

$$
\vec{t}_{t}=\vec{a}_{t}+\vec{b}_{t}+\vec{c}_{t}+\vec{d}_{t}
$$

caractérise la déformation (prise ici au sens de non-centrage) due à la croissance durant l'année t considérée.

Notons $e_{t}$ l'amplitude de cette déformation :

$$
e_{t}=\left\|\vec{l}_{t}\right\|=\left\|\vec{a}_{t}+\vec{b}_{t}+\vec{c}_{t}+\vec{d}_{t}\right\|
$$

La direction de la déformation est donnée par :

$$
\sin \hat{\alpha}_{t}=\sin \left(\vec{i}, \vec{l}_{t}\right)=\left(\left\|\vec{b}_{t}\right\|-\left\|\vec{d}_{t}\right\|\right) / e_{t}
$$
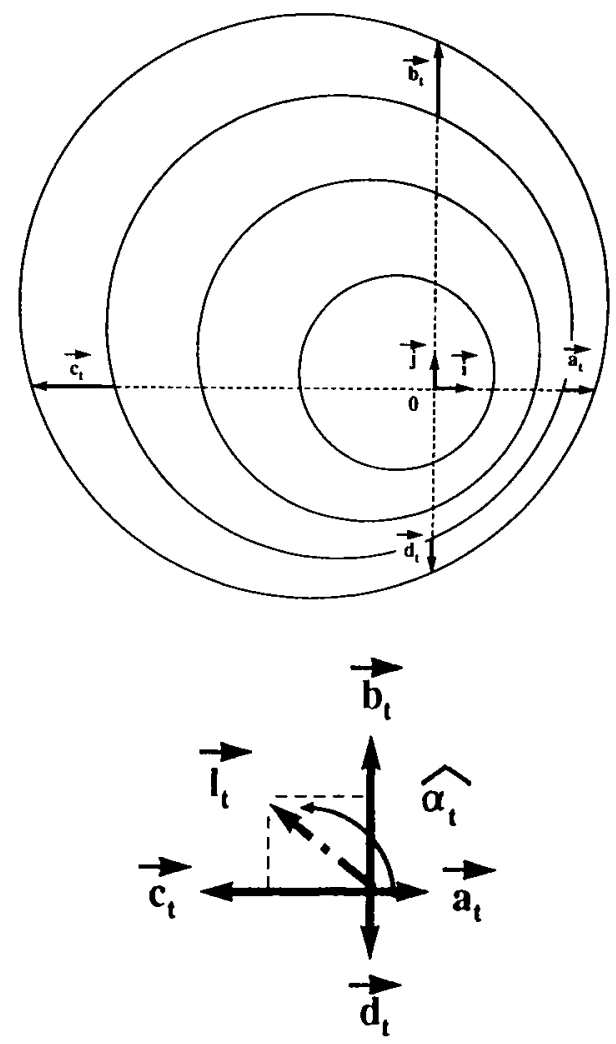

Fig 5. Construction du vecteur $\vec{l}_{t}$ relatif à la croissance d'une section durant une année t.

Si la procédure précédente est appliquée depuis l'origine de l'arbre où le centrage de la section est évidemment parfait, il est possible de connaître la déformation $\overrightarrow{\mathrm{L}}_{\mathrm{T}}$, liée à la croissance depuis l'origine jusqu'à un âge $\mathrm{T}$ donné.

$$
\vec{L}_{T}=\sum_{t=1}^{T} \vec{I}_{t}
$$

En cohérence avec la notation utilisée précédemment, nous avons : 


$$
\begin{aligned}
& \vec{A}_{T}=\sum_{t=1}^{T} \vec{a}_{t}, \quad \vec{B}_{T}=\sum_{t=1}^{T} \vec{b}_{t}, \quad \vec{C}_{T}=\sum_{t=1}^{T} \vec{c}_{t}, \\
& \vec{D}_{T}=\sum_{t=1}^{T} \vec{d}_{t}, \quad \vec{L}_{T}=\sum_{t=1}^{T} \vec{l}_{t} \\
& E_{T}=\left\|\vec{L}_{T}\right\|=\left\|\vec{A}_{T}+\vec{B}_{T}+\vec{C}_{T}+\vec{D}_{T}\right\| \\
& \text { et } \sin \hat{\beta}_{T}=\frac{\left\|\vec{B}_{T}\right\|-\left\|\vec{D}_{T}\right\|}{E_{T}}
\end{aligned}
$$

Ce type de démarche permet d'obtenir des représentations graphiques comme celle présentée sur la figure 6 .
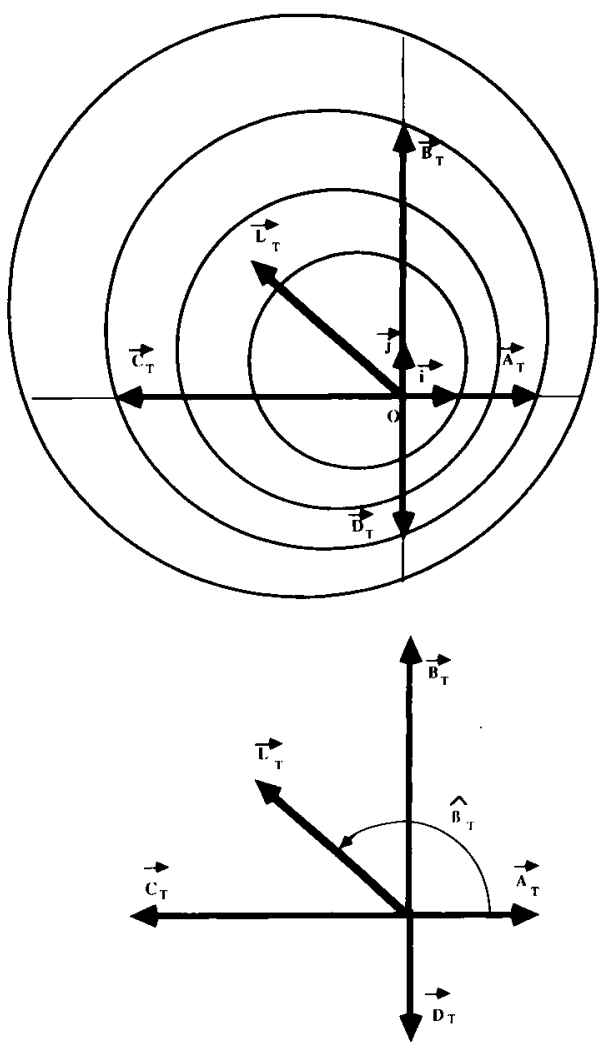

Fig 6. Construction du vecteur $\vec{L}_{T}$ relatif à la croissance d'une section depuis l'origine jusqu'à l'année T.

\section{Amélioration de la méthode proposée}

En privilégiant l'étude des accroissements, Marutani et al négligent la définition et l'évolution du centre géométrique. Par ailleurs la méthode qu'ils proposent se limite à la mesure de 2 diamètres; or, il a été précisé précédemment que 2 directions ne permettent pas de rendre compte, dans la plupart des cas, de l'irrégularité d'une section, ni de rapprocher les observations réalisées sur différents niveaux d'une même tige, ni celles effectuées sur un même niveau pour différentes tiges.

C'est pourquoi les améliorations qui vont suivre sont proposées.

\section{Définition et évolution du centre géométrique d'une section}

En restant dans le contexte proposé par Marutani et al, le vecteur $\overrightarrow{\mathrm{L}}_{\mathrm{T}} / 4$ "caractérise" le centre géométrique $G_{T}$ de la section à l'âge $T$, et l'évolution de ce centre est décrit par une trajectoire dont les $\overrightarrow{l_{t}} / 4$ sont les composantes, puisque

$1 / 4 \vec{L}_{T}=1 / 4 \sum_{t=1}^{T} \vec{l}_{t} \quad$ (fig 7 et 8 ).

Remarque : par centre géométrique, on entend celui défini en fonction des croissances radiales relevées sur les 2 diamètres de référence. En fait, le centre défini comme géométrique n'est qu'une estimation d'autant moins précise que la forme de la section s'écarte d'un disque parfait et que le décentrage est prononcé. Cette estimation peut être améliorée en prenant un nombre plus élevé de diamètres.

\section{Accroissement annuel sur $\mathbf{2 N}$ diamètres de référence}

Le raisonnement tenu précédemment pour 2 diamètres peut être étendu à l'utilisation 

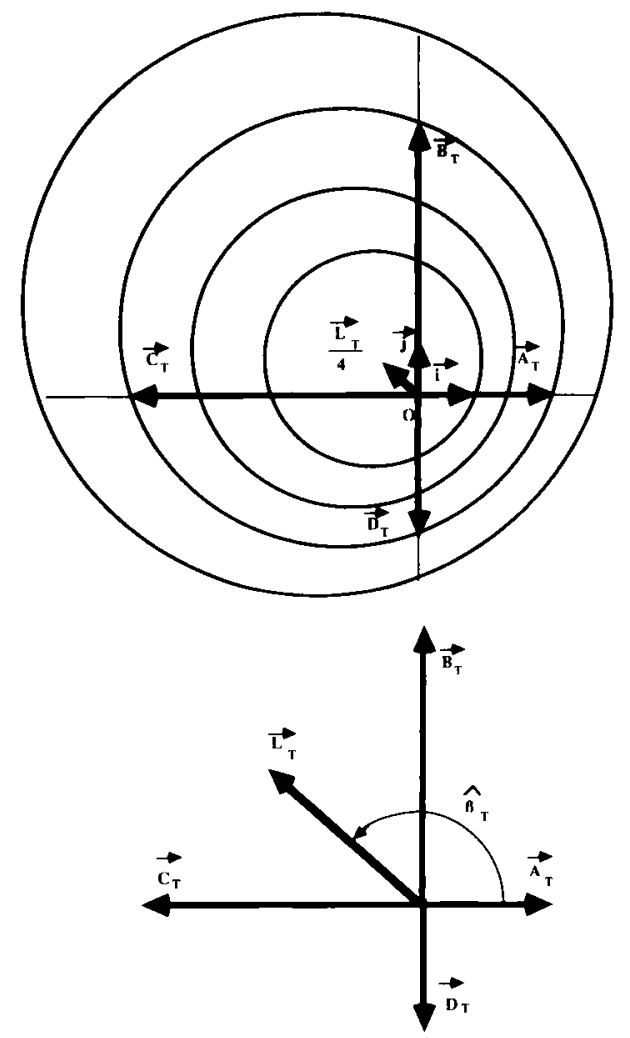

Fig 7. Construction du vecteur $\overrightarrow{\mathbf{L}}_{\tau} / 4$ permettant de définir le centre géométrique d'une section à l'année T.

de $2 \mathrm{~N}$ diamètres, 2 rayons consécutifs étant séparés de $\pi / 2 \mathrm{~N}$ radian.

En effet, à chaque couple $c$ de diamètres $(c=1$ à $\mathrm{N})$ perpendiculaires va correspondre:

- une déformation $\vec{l}_{t, c}$ liée à la croissance durant l'année $t$, d'amplitude $\mathrm{e}_{(\mathrm{t}, \mathrm{c})}$ et faisant un angle $\hat{\alpha}_{(\mathrm{t}, \mathrm{c})}$ avec $\overrightarrow{\mathrm{i}}$;

- une déformation $\vec{L}_{T, c}$ liée à la croissance depuis l'origine jusqu'à une année donnée $T$, d'amplitude $E_{T, c}$ et faisant un angle $\beta_{T, c}$ avec $\vec{i}$.

II sera donc possible de définir (figs 9 et 10) :

$$
\begin{aligned}
& \vec{l}_{t}=\sum_{c=1}^{N} \vec{l}_{(t, c)} \\
& \vec{L}_{T}=\sum_{c=1}^{N} \vec{L}_{(T, c)} \quad \operatorname{avec} \vec{L}_{(T, c)}=\sum_{t=1}^{T} \vec{l}_{(t, c)} \\
& \hat{\alpha}_{t}=\operatorname{angle}\left(\vec{i}, \vec{l}_{t}\right) \\
& \hat{\beta}_{T}=\text { angle }\left(\vec{i}, \vec{L}_{T}\right)
\end{aligned}
$$

\section{Approche analytique}

Analytiquement parlant, il est possible de définir les coordonnées des vecteurs $\vec{I}_{t}, \vec{L}_{T}$ et celles du centre géométrique GT dans le repère $(0, \vec{i}, \vec{j})$.

Notons $r_{(T, m)}$ la longueur $d u m^{e}$ rayon (celui qui fait un angle $(m-1) \pi / 2 N$ avec $\vec{i}$ ) à l'âge $T$, les coordonnées des vecteurs $\vec{l}_{t}$ et $\vec{L}_{T}$ et de $G_{T}$ ont pour valeur :

$$
\begin{aligned}
& x_{t}=\sum_{m=1}^{4 N}\left(r_{(T, m)}-r_{(T-1, m)} \cos (m-1) \frac{\pi}{2 N}\right. \\
& y_{t}=\sum_{m=1}^{4 N}\left(r_{(T, m)}-r_{(T-1, m)} \sin (m-1) \frac{\pi}{2 N}\right.
\end{aligned}
$$$$
\overrightarrow{I_{t}}=
$$

$$
\begin{gathered}
\mathrm{X}_{T}=\sum_{m=1}^{4 N} r_{(T, m)} \cos (m-1) \frac{\pi}{2 N} \\
\overrightarrow{\mathrm{L}}_{T=} \mathrm{Y}_{\mathrm{t}}=\sum_{m=1}^{4 N} r_{(T, m)} \sin (m-1) \frac{\pi}{2 N} \\
\mathrm{G}_{\mathrm{GT}}=\frac{1}{4 N} \sum_{m=1}^{4 N} r_{(T, m)} \cos (m-1) \frac{\pi}{2 N} \\
Y_{G T}=\frac{1}{4 N} \sum_{m=1}^{4 N} r_{(T, m)} \sin (m-1) \frac{\pi}{2 N}
\end{gathered}
$$



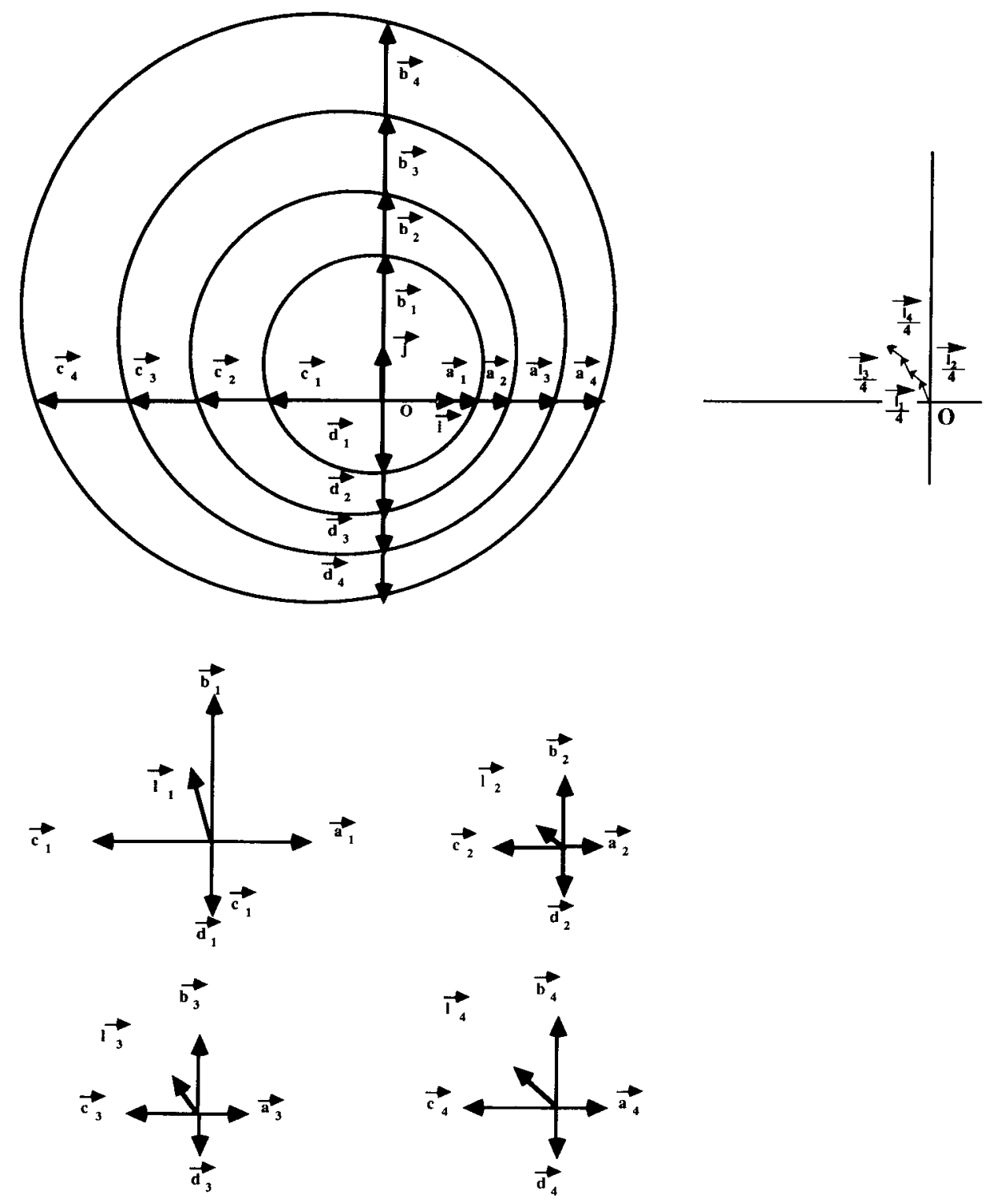

Fig 8. Évolution du centre géométrique d'une section depuis l'origine. 

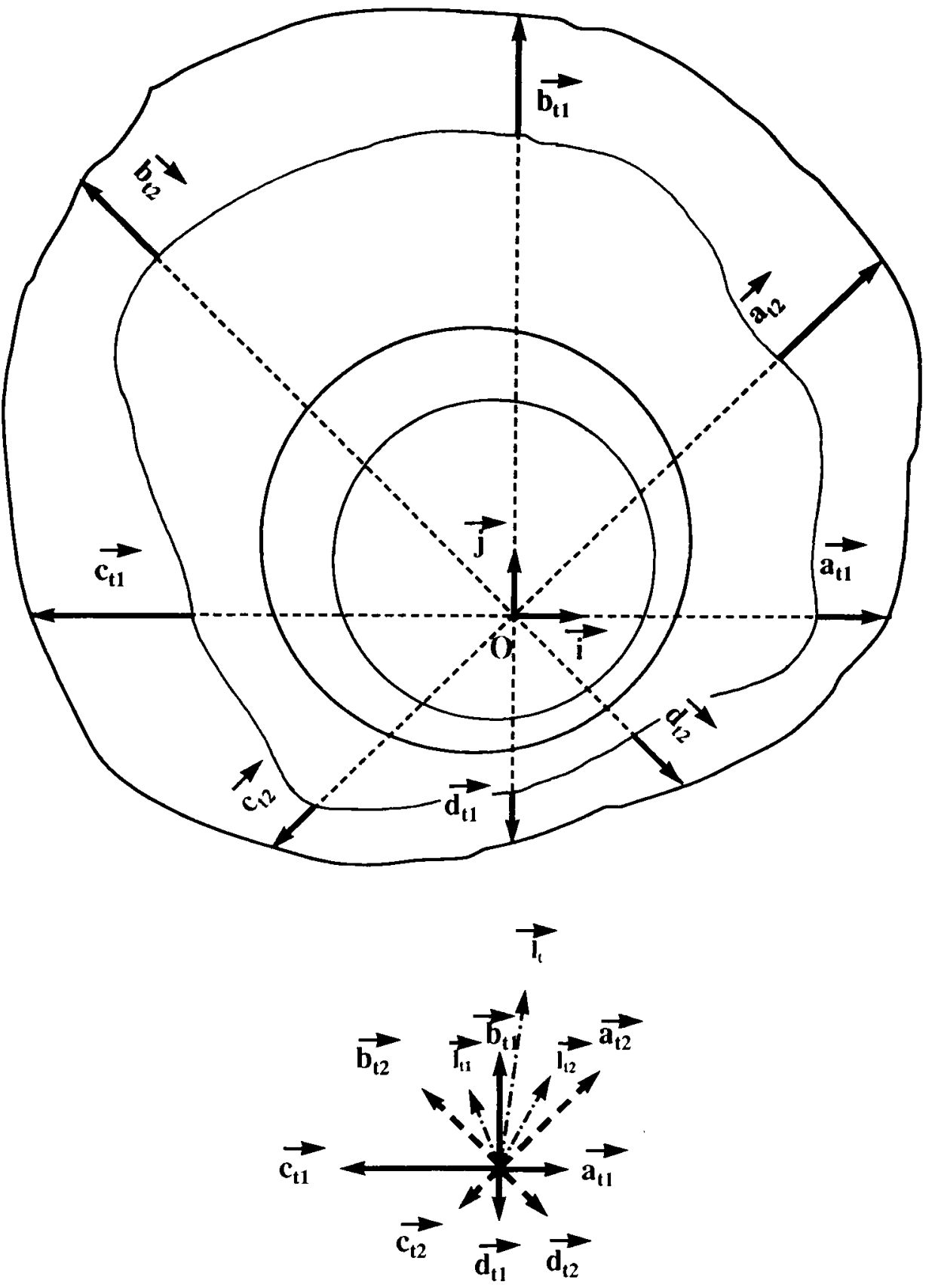

Fig 9. Construction du vecteur $\overrightarrow{l_{t}}$ moyen correspondant à la croissance d'une section pour une année t. Exemple avec l'emploi de 4 diamètres. 

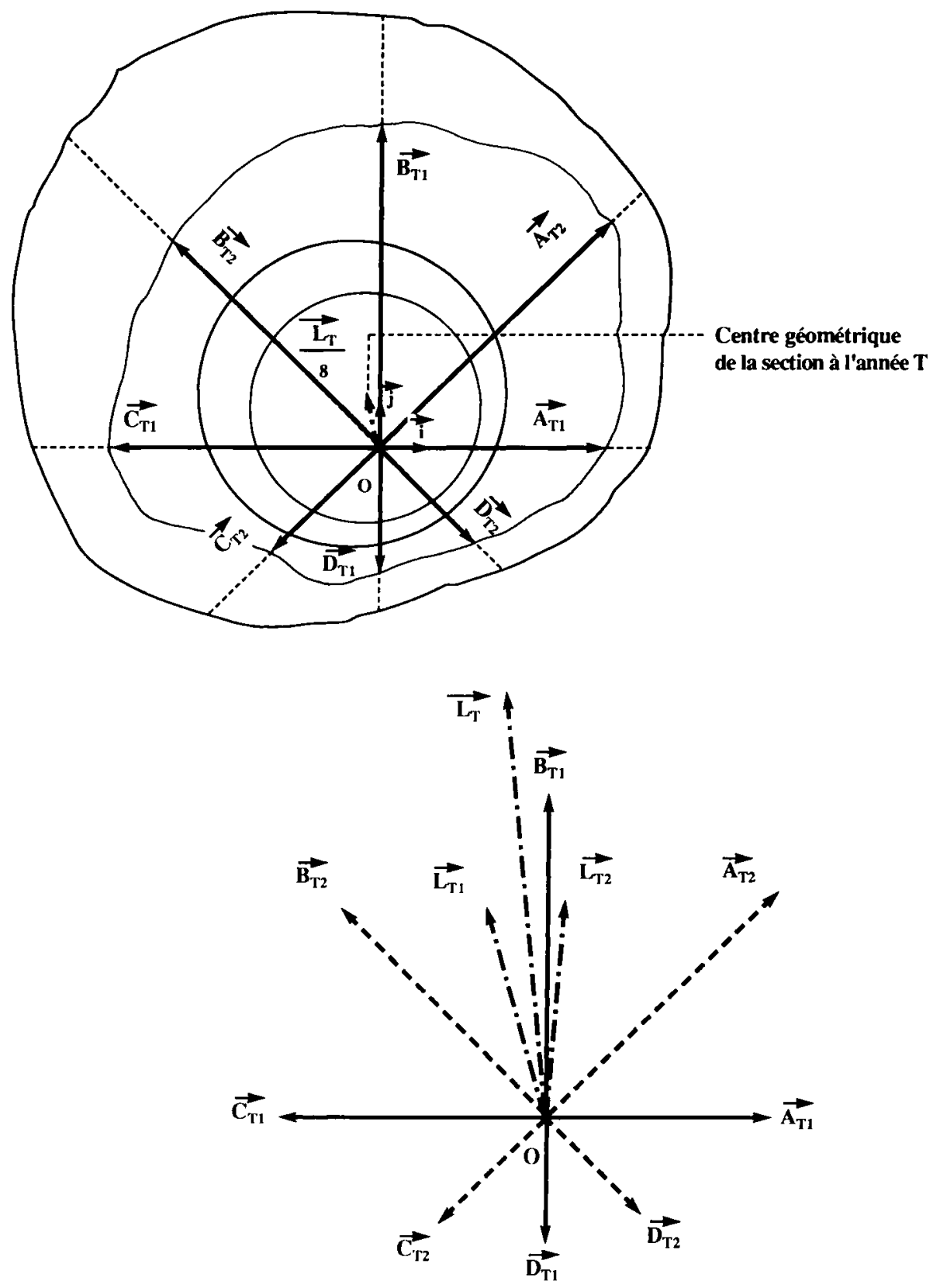

Fig 10. Construction du vecteur $\vec{L}_{T}$ et localisation du centre géornétrique d'une section à l'année $T$. 
Le centre géométrique ainsi défini est donc le centre de gravité des points de coordonnées:

$$
\left(r_{(T, m)} \cos (m-1) \frac{\pi}{2 N}, r_{(T, m)} \sin (m-1) \frac{\pi}{2 N}\right)
$$

définis pour $m$ variant de 1 à $4 \mathrm{~N}$.

À titre d'exemple, l'évolution des coordonnées du centre géométrique de la section représentée à la figure $\uparrow$ est donnée dans le tableau I.

\section{Rapprochement des observations faites à un même niveau pour différentes tiges}

II est essentiel de rappeler que l'évolution du centre géométrique s'entend dans un système de référence donné et que les excentricités relevées sur 2 arbres ne peuvent pas être comparées directement.

La figure 11 met en évidence ce problème : soit 2 arbres qui ont, à un niveau donné, exactement la même forme de section (ici un disque parfait), mais qui présentent des excentricités différentes. À partir d'une référence commune - par exemple le milieu d'une face du tronc en se repé-

Tableau I. Évolution des coordonnées du centre géométrique de la section représentée sur la figure 10.

\begin{tabular}{ccc}
\hline $\begin{array}{c}\text { Année } \\
\text { de } \\
\text { croissance }\end{array}$ & $\begin{array}{c}\text { Coordonnées } \\
(\text { en } \| \text { ii) }\end{array}$ & $\begin{array}{c}\text { Coordonnées } Y_{G} \\
\text { (en }\|j\| I /\end{array}$ \\
\hline & & \\
1 & $-0,1821$ & 0,4931 \\
2 & $-0,3376$ & 0,6486 \\
3 & $-0,1186$ & 1,2788 \\
\hline
\end{tabular}

rant sur la ligne de plantation ${ }^{*}$ - il est possible de définir les repères $(0, \vec{i}, \vec{j})$ et $\left(o^{\prime}, \vec{i}^{\prime}, \overrightarrow{j^{\prime}}\right)$. Pour une même évolution du centre géométrique durant une année donnée, ici la dernière à titre d'exemple, il apparait que le vecteur $\overrightarrow{l_{t}} / 4$ est, logiquement, orienté différemment par rapport à $\vec{i}$ et à $\overrightarrow{i^{\prime}}$.

En fait, pour pouvoir comparer directement l'évolution des centres géométriques, il faudrait pouvoir "superposer» les 2 repères, ce qui revient à connaître l'angle $\hat{\theta}$, $\operatorname{car} \hat{\alpha}_{t}^{\prime}=\hat{\alpha}_{t}-\hat{\theta}$.

$$
\begin{aligned}
& \text { En effet, } \hat{\gamma}=\hat{\epsilon} \text { et } \hat{\pi} / 2=\hat{\delta}+\hat{\gamma}=\hat{\delta}^{\prime}+\hat{\gamma} \\
& \hat{\gamma^{\prime}}=\hat{\epsilon}+\hat{\theta} \\
& \text { d'où } \hat{\delta}-\hat{\delta}^{\prime}=\hat{\theta} \text {. } \\
& \text { Or } \hat{\alpha}_{\mathrm{t}}-\hat{\alpha}_{\mathrm{t}}^{\prime}=(\hat{\pi}+\hat{\theta})-\left(\hat{\pi}+\hat{\delta}^{\prime}\right) \\
& ==>\hat{\alpha}_{t}-\hat{\alpha}_{t}^{\prime}=\hat{\theta}
\end{aligned}
$$

Remarque : la démonstration serait équivalente quel que soit l'endroit de la section où se situent 0 et 0 '.

Pour connaître l'angle $\hat{\theta}$, il suffit de pouvoir calculer les angles $(\vec{j}, \overrightarrow{S N})$ et $\left(\overrightarrow{j^{\prime}}, \overrightarrow{S N}\right)$, $S N$ représentant une direction de référence (sud/nord par exemple ou, comme il a été suggéré précédemment, la direction de la ligne de plantation).

L'utilisation de cette dernière direction comme référence est dans la pratique souvent à recommander, car elle permet de repérer plus facilement sur le terrain la position des arbres voisins de l'arbre sujet, cela dans le but de tenter d'évaluer l'influence de ceux-ci sur l'excentricité étudiée.

\section{Rapprochement des observations faites à différents niveaux d'une même tige}

Ce qui vient d'être avancé au paragraphe "Rapprochement des observations faites à un même niveau(...)" peut être repris, à la

\footnotetext{
* Toutes les lignes étant supposées orientées dans la même direction.
} 


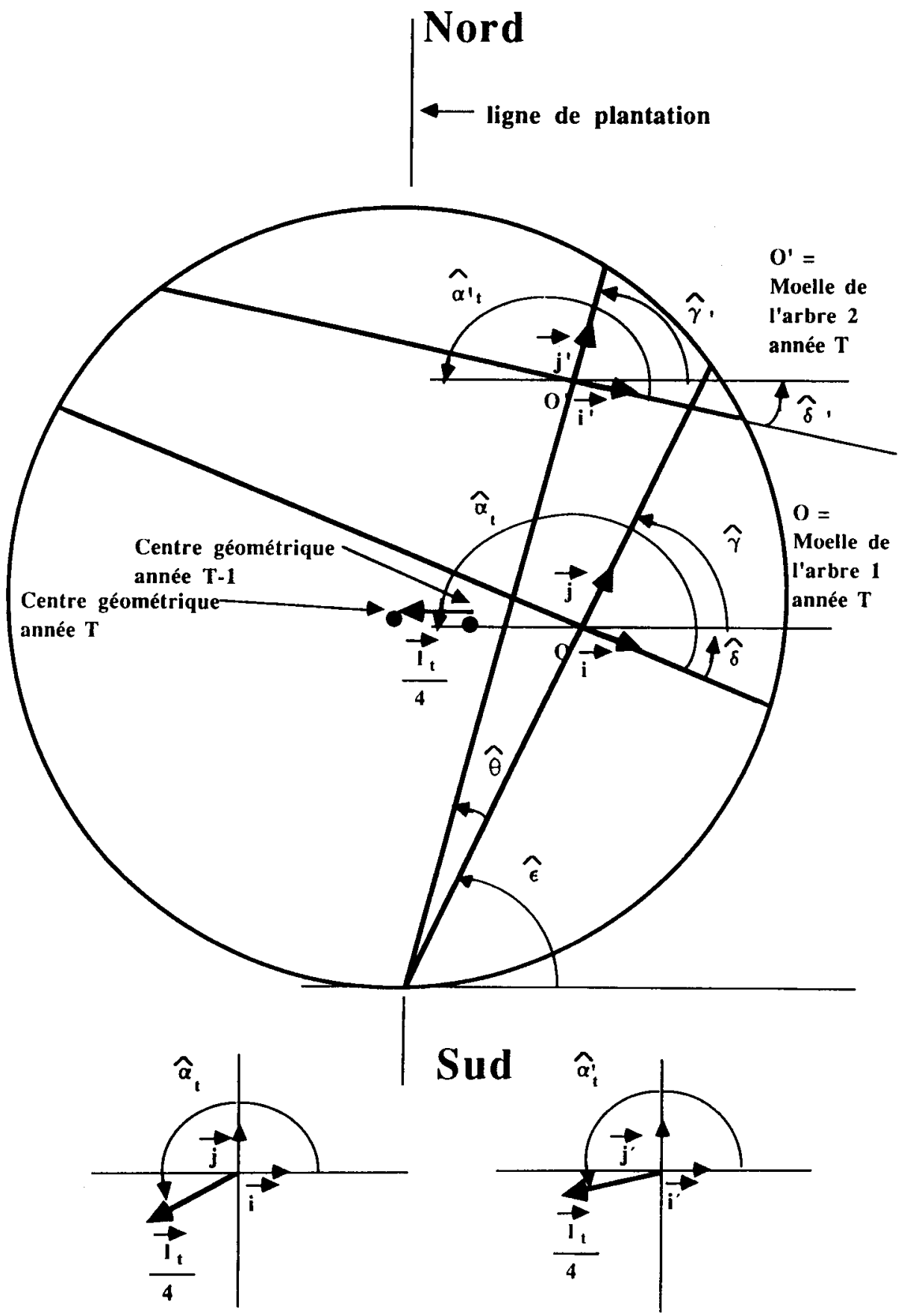

Fig 11. Représentation d'une même excentricité d'une section due à la croissance de l'année $T$ depuis 2 repères différents. 
différence près que plus les rondelles sont prélevées à un niveau élevé dans l'arbre, et moins le nombre d'années de croissance prises en compte est important.

L'évolution du centre géométrique ne concernera donc pas forcément des intervalies de temps de même amplitude selon les niveaux, et il faudra en tenir compte dans les rapprochements des différentes excentricités relevées.

Remarque : puisque l'importance d'une direction de référence est apparue, il semble intéressant de proposer une méthode qui permette de matérialiser cette direction avec assez de précision.

On considère tout d'abord une direction $\vec{D}$ donnée (la ligne de plantation, par exemple). À toute génératrice extérieure du tronc $G$ correspond une "génératrice opposée» G' obtenue comme l'interşection du tronc et des droites de direction $\vec{D}$ s'appuyant sur $G$. La position d'une rondelle est déterminée par la donnée des 2 points opposés situés sur $\mathrm{G}$ et $\mathrm{G}^{\prime}$. On peut obtenir ces points en utilisant un appareil du type de celui présenté sur la figure 12,

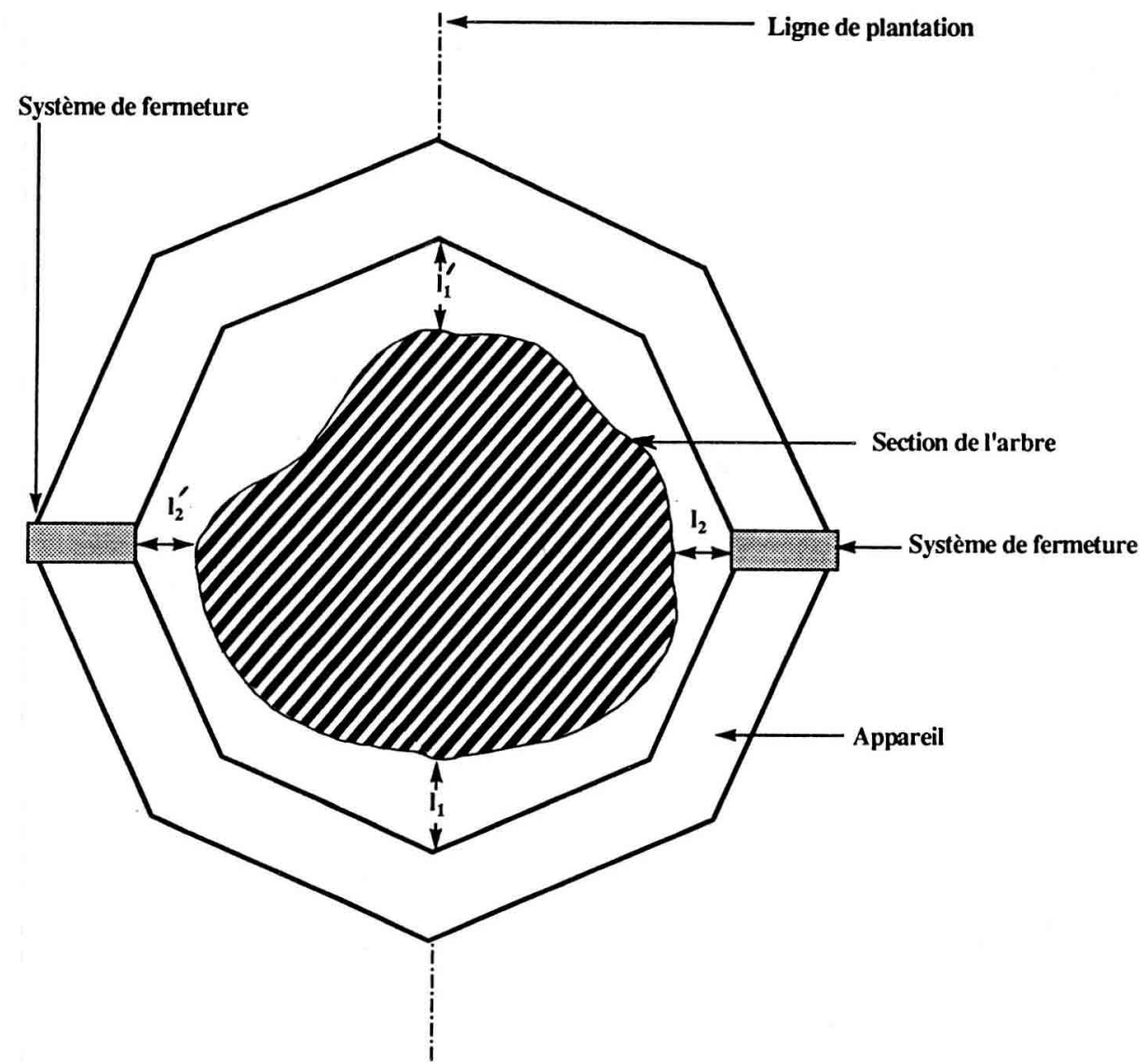

Fig 12. Appareil permettant de placer 2 marques à $180^{\circ}$ sur un tronc en s'appuyant sur la ligne de plantation. Vue de dessus. 
réglé de telle façon que $\vec{l}_{1}, \vec{l}_{1}$ et $D$ aient même direction et $\vec{l}_{2}^{\prime}=\vec{I}_{2}$.

II est impératif de réaliser cette opération avant que les différentes rondelles ne soient prélevées. En effet, si cela n'est pas le cas, le fait que les différentes rondelles soient susceptibles d'être mobiles, bien que d'une manière limitée, autour d'un axe pivot $^{\star}$ - la génératrice de référence conduit inévitablement à une imprécision dans l'établissement de la direction de référence.

\section{Indices supplémentaires}

II est possible, pour caractériser au mieux la déformation de la section, d'introduire un pourcentage de distorsion (Marutani et al, 1987).

Celui-ci permet de rapprocher l'évolution de l'excentricité d'une section et l'accroissement radial de cette dernière, en tenant compte, par exemple, du fait que 2 sections peuvent présenter des accroissements radiaux moyens différents, mais une évolution comparable de leur excentricité.

D'une manière générale, cet indice devrait permettre de mieux rapprocher les évolutions des excentricités observées sur des arbres de vigueur différente, toutes choses étant égales par ailleurs (environnement...). Ce pourcentage est défini comme suit :

- durant une année t $\quad p d=\frac{e_{t}}{\overline{\Delta r_{t}}}$

avec $e_{t}$ la déformation liée à la croissance durant l'année t telle que définie au paragraphe "Définition et évolution du centre géométrique d'une section». et $\quad \overline{\Delta r}_{\mathrm{t}}=\sum_{\mathrm{m}-1}^{4 \mathrm{~N}} \frac{\|\overrightarrow{\mathrm{a}(\mathrm{m}, \mathrm{t})}\|}{4 \mathrm{~N}}$

$\overrightarrow{\mathbf{a}}_{(\mathrm{m}, \mathrm{t})}$ vecteur représentant l'accroissement durant l'année $t$ relevé sur le $m^{e}$ rayon de référence.

- depuis l'origine jusqu'à une année T

$$
\mathrm{PD}=\frac{\mathrm{E}_{\mathrm{T}}}{\overline{\Delta \mathrm{R}_{\mathrm{T}}}}
$$

avec $E_{T}$ la déformation de la section liée à la croissance depuis l'origine jusqu'à une année $\mathrm{T}$ donnée, comme définie au paragraphie «Principes de la méthode proposée par Marutani et al’s,

et

$$
\overline{\Delta R_{t}}=\Sigma_{t=1}^{T} \overline{\Delta r_{t}}
$$

De la même façon, il serait possible d'employer pour caractériser l'excentricité d'une section et son évolution

$$
\frac{\vec{l}_{t}}{4 \mathrm{~N}{\overline{\Delta R_{t}}}_{n}}, \frac{\vec{L}_{T}}{4 \mathrm{~N} \overline{\Delta R}_{T}} .
$$

\section{MÉPLAT}

Pour caractériser le méplat d'une section, il est possible d'utiliser l'indice suivant :

$$
\begin{gathered}
\begin{array}{c}
\text { diamètre perpendiculaire } \\
\text { au plus grand diamètre }
\end{array} \\
\text { plus grand diamètre }
\end{gathered}
$$

comme l'ont préconisé Polge et Illy (1967).

\footnotetext{
* En effet, faire correspondre d'une façon sûre les différentes rondelles les unes par rapport aux autres n'est pas toujours possible (forme de la section variant sensiblement entre 2 niveaux).
} 
On peut aussi définir "l'angle du méplat» comme l'angle que fait le plus grand diamètre avec une direction de référence définie au paragraphe «Rapprochement des observations (...)". L'intérêt de ce paramètre est de pouvoir quantifier une certaine évolution du méplat.

Cependant, il faut remarquer que le diamètre peut indifféremment être défini (fig 13) comme passant :

- par le centre géométrique défini au paragraphe "Définition et évolution du centre géométrique d'une section";

- par la moelle de l'arbre.

\section{Diamètre passant par le centre géométrique}

II est certain que la première définition semble à retenir car elle rend mieux compte a priori du phénomène.

Cependant, il est nécessaire alors, pour chacun des centres trouvés, de se reporter à la rondelle, ce qui peut poser des problèmes pratiques : laps de temps pouvant être non négligeable entre la lecture des cernes et l'interprétation des résultats, d'où la nécessité d'un stockage adéquat pour que les rondelles ne fendent pas, qui n'est

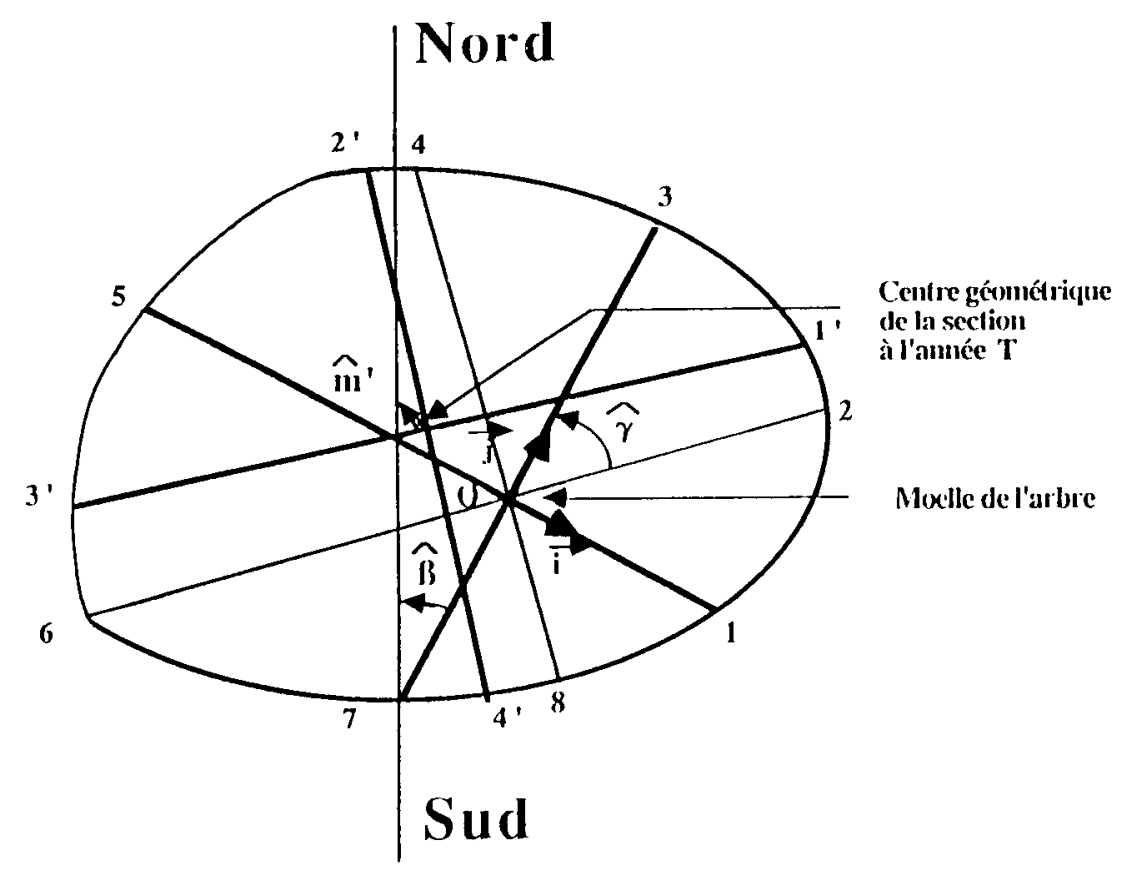

Fig 13. Exemple de calcul du méplat d'une section en faisant passer le plus grand diamètre par la moelle de l'arbre ou par le centre géométrique. Emploi de 8 rayons.

Calcul du méplat de la section : a) par la moelle de l'arbre :

$M=\frac{[4-8]}{[2-6]}=0,69$; orientement : $m=\beta+\gamma=75^{\circ}$;

b) par le centre géométrique :

$M^{\prime}=\frac{\left[2^{\prime}-4^{\prime}\right]}{\left[1^{\prime}-3^{\prime}\right]}=0,72$; orientement : $m^{\prime}=78^{\circ}$. 
pas toujours à disposition (chambre froide).

\section{Diamètre passant par la moelle de l'arbre}

L'utilisation de diamètres passant par la moelle de l'arbre permet de calculer directement $\mathrm{M}$ pour chacune des années considérées. De plus, les angles que font les différents diamètres avec la direction de référence étant fixes et mesurés lors de la lecture des cernes, l'évolution de la direction du méplat pourra être facilement estimée.

Même si cette deuxième méthode est certainement moins satisfaisante, elle peut tout de même paraître acceptable si le nombre de rayons adoptés est assez élevé ( $\geq 16)$.

\section{CONCLUSION}

La méthode proposée tente de rendre compte de la déformation de la section des tiges et de son évolution au cours du temps.

Elle prend en compte l'excentricité des tiges (décentrage observé) et le méplat caractérisant l'aplatissement d'une section.

La lecture de cernes est nécessaire, le nombre de 8 rayons semblant un strict minimum pour caractériser d'une manière pas trop grossière le phénomène.

Aussi la méthode est-elle contraignante quand les accroissements radiaux ne sont pas mesurés automatiquement, mais nécessitent des moyens manuels (lecture au double décimètre...).

Cependant, l'utilisation de techniques plus performantes (digitalisation des cernes...) devrait pouvoir rendre plus aisée dans l'avenir l'application d'un tel type de méthode.

\section{RÉFÉRENCES}

Armitage FB, Burley J (1980) Pinus kesiya. Trop For Paper 9, Commonwealth Institute, $199 \mathrm{p}$

Biging G, Wensel LC (1988) The effects of eccentricity on the estimation of basal area and basal area increment of coniferous trees. For Sci 34 (3), 621-633

Boissieras A (1984) Recherche de prédicteurs juvéniles de l'aptitude génétique à la croissance en volume chez le pin maritime. Détermination de l'âge optimal pour la sélection. Mémoire de stage option "forêt", École forestière de Meymac, INRA, département forêt Bordeaux, $32 \mathrm{p}$

Cailliez F (1980) Estimation des volumes et accroissement des peuplements forestiers. Vol 1. Estimation des volumes. Étude FAOForêts22/1, $99 \mathrm{p}$

Coue JC, Guitard D, Bailleres H (1990) Stabilité dimensionnelle des contreplaqués à base de bois d'essences différentes. Utilisation de pin maritime. Compte rendu de fin d'étude d'une recherche financée par le ministère de la Recherche et de la Technologie, décision d'aide $N^{\circ} 88-A-0339,129 p$

Demaerschalk JP, Kozak A (1977) The whole bole system: a conditionned dual equation system for precise prediction of tree profiles. Can J For Res 7, 488-497

Détienne $P$ (1976) Recherche et nature du bois de tension dans quelques bois tropicaux. Note CTFT, $46 \mathrm{p}$

Farrar JL (1961) Longitudinal variation in the thickness of the annual ring. Forestry Chronicle 349, 323-330

Farrar RM Jr (1987) Stem profile functions for predicting multiple-product volumes in natural longleaf pines. South J Appl For 11(3), 161167

Fournier M (1989) Mécanique de l'arbre sur pied : maturation, poids propre, contraintes climatiques dans la tige standard. Thèse de I'Institut Polytechnique de Lorraine en sciences du bois, 16 novembre 1989, 257 p

Gordon A, Graham JD (1986) Changes in Pinus radiata stem form in response to nitrogen and phosphorus fertiliser. $N Z J$ For Sci 16 (1), 41-54 
Kellog RM, Barber FJ (1981) Stem eccentricity in coastal western hemlock. Can J For Res $11,714-718$

Kozak A (1988) A variable exponent taper equation. Can J For Res 18, 1363-1368

Larson PH (1963) Stem form development of forest trees. For Sci Monogr 5, $41 \mathrm{p}$

Lowell KE (1986) A flexible polynomial taper equation and its suitability for estimating stem profiles and volumes of fertilized and unfertilized Radiata pine trees. Aust For Res $16,165-174$

Marutani T, Aragami K, Yuruki T (1987) A method for analysis of annual rings influenced by environment (II). Bull Kyushu Univ For 57 , 1-8

Mc Clure JP, Czaplewski RL (1986) Compatible taper equation for loblolly pine. Can J For Res 16, 1272-1277

M'Hirit O, Postaire JG (1983) Analyse de la forme des tiges du cèdre du Maroc. Applica- tion à la détermination des courbes de profil. Ann Sci For 40(4), 355-372

Mitchell KJ (1975) Dynamics and simulated yield of Douglas fir. For Sci Monogr 17, supplement to For Sci 21(4), $40 \mathrm{p}$

Mitchell KJ, Kellog RM (1972) Distribution of area increment over the bole of fertilized Douglas fir. Can J For Res 2, 95-97

Monserud RA (1979) Relations between inside and outside bark diameter at breast height for Douglas fir in northem Idaho and northwestern Montana. USDA For Sen Research note Int 266, $8 \mathrm{p}$

Pawsey CK (1966) Lean and eccentricity in Pinus radiata (D Don) in the southeast of South Australia. Aust For Res 2 (3), 22-35

Polge H, Illy G (1967) Observations sur lanisotropie du pin maritime des Landes. Ann Sci For 24 (3), 205-231

Williamson RL (1975) Out of roundness in Douglas fir stems. For Sci 21 (4), 365-370 